\title{
A NEW METHOD FOR ESTIMATING EFFECTS OF VISUAL FIELD LOSS IN A PANORAMIC DRIVING ENVIRONMENT
}

\author{
David E. Anderson ${ }^{1 *}$, Deepta A. Ghate ${ }^{2}$, Sachin Kedar ${ }^{1,2}$, Matthew Rizzo ${ }^{1}$ \\ ${ }^{1}$ Department of Neurological Sciences, College of Medicine \\ ${ }^{2}$ Truhlsen Eye Institute, College of Medicine \\ University of Nebraska Medical Center \\ Omaha, NE, USA \\ *Email: david.anderson@unmc.edu
}

\begin{abstract}
Summary: Glaucoma is a key cause of peripheral visual field loss and increases risk of a vehicle crash. Patients may be unaware of their visual loss and of hazards in the driving panorama. Standard clinical automated perimetry, the "gold standard" for monitoring glaucoma progression, lacks external validity to evaluate functional effect of visual field loss in driving environments. We developed and piloted a new technique to study the effects of glaucoma in a panoramic $\left(290^{\circ}\right.$ forward FOV) simulated driving environment. Preliminary results in 11 drivers ( 7 with glaucoma and 4 with suspected glaucoma): (1) demonstrate the relationship between standard clinical perimetry and driving simulator visual fields, (2) replicate clinical evidence of glaucoma-related peripheral visual field loss, and (3) show added visual field loss due to visual occlusion by in-cab geometry.
\end{abstract}

\section{INTRODUCTION}

Glaucoma is a major public health concern, affecting $2 \%$ of the U.S. population over the age of 40 (Friedman et al., 2004). This potentially blinding optic neuropathy is characterized by peripheral vision loss. Visual field defects in glaucoma affects driving performance (Crabb et al., 2013). U.S. drivers with glaucoma had 1.65 times greater MVC rates compared to the general population, with severe visual field loss being the highest ranked risk factor for MVC (Kwon, 2016). A case-control study showed a 3.6-fold increased risk of an injurious crash in glaucoma. Peripheral (30-60 degrees) visual field defects greater than $10 \mathrm{~dB}$ led to a 2.4 times increased risk (Owsley et al., 1998). Glaucoma patients were 6 times more likely to need a critical intervention from a driving instructor compared to controls, particularly for unnoticed pedestrian (Haymes, 2008).

Glaucoma patient symptoms may provide clues to mechanisms of increased driving risk. Some glaucoma patients report "needing more light" or "blurry vision" (Hu et al., 2014). In a forced choice experiment, patients with bilateral glaucoma described their visual experience as "blurred" (54\%) or "normal" (26\%) when viewing a naturalistic display (Crabb et al., 2013); contradicting what is commonly taught, none reported a black spot or hole in vision. One hypothesis suggests glaucoma patients "fill in" their visual field defects, as in perceptual completion of physiologic blind spot (where the optic nerve enters the back of retina). Evidence from physiologic, artificial, and retinal scotomas (areas of vision loss surrounded by preserved vision) suggest drivers with glaucoma do not appreciate the role of their vision impairment in their failure to perceive critical objects or events (Hoste, 2003; Figure 1). 


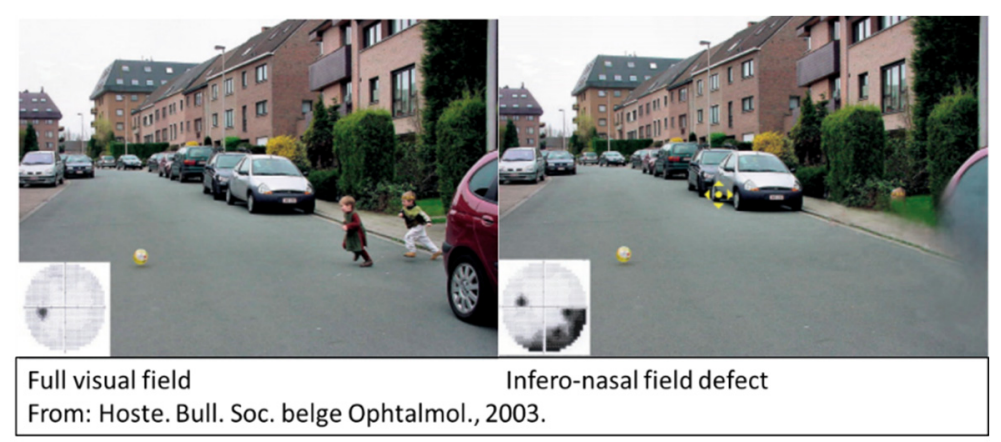

Figure 1. Simulating perceptual experiences of full visual fields and visual field defects

To study the effects of glaucomatous visual field loss on driving, it is critical to measure visual fields in an immersive driving environment. While standard clinical automated perimetry for the assessment of visual field loss - such as the Humphrey visual field (HVF) test - are essential for monitoring disease progression, their unknown external validity limits extrapolation to real world effects. This study sought to adapt the HVF into a full-sized high-fidelity driving simulator. Our goals were to: (1) better understand the relationship between standard clinical measures of visual field loss and effective fields of view in naturalistic settings; (2) replicate evidence of glaucomarelated peripheral visual field loss in a driving simulator visual field (DSVF) test; (3) evaluate added effects of visual occlusion due by vehicle geometry.

\section{METHODS}

Subjects. Eleven drivers (45\% women), including 7 with glaucoma and 4 with suspected glaucoma (diagnosed using standard clinical criteria), participated. Mean ages were $66.1 \pm 17.2$ (SD) and $65.5 \pm 10.1$, for glaucoma patients and suspects, respectively. All subjects were recruited from the Truhlsen Eye Institute at the University of Nebraska Medical Center (UNMC) in Omaha, NE. Glaucoma suspects had normal visual fields; glaucoma patients had a range of visual field defects. All had corrected visual acuity of at least 20/40 in each eye and were experienced visual field takers with at least three stable (reliability indices better than 20\%) HVFs over the last 2 years. Subjects were excluded if they had a diagnosis of neurological disorder or cognitive impairment. This study was approved by the UNMC IRB.

Visual Field Mapping. Visual field testing was performed in the ophthalmology clinic using the 30-2 or the 24-2 SITA standard strategy in automated perimetry (Humphrey Field Analyzer II-i, Carl Zeiss Meditec Inc) as part of routine clinical care prior to recruitment for the study.

Driving Simulator. SENSEI (Simulator for Ergonomics, Neuroscience, Safety Engineering and Innovation), a DriveSafety (Salt Lake City, UT) RS-600 Research Simulator, is a fully integrated, high performance, high fidelity driving simulation system with an authentic automotive cab designed for use in ground vehicle design and driving safety research applications (Figure 2a). Coupled with HyperDrive advanced scene and scenario authoring tools, the SENSEI simulation system provides an ideal environment to create purpose-built driving scenarios for a wide range of research studies. The RS-600 provides an out-the-window display environment of 290 degrees with 7 Ultra-HD (3840x2160 each) curved LED displays, a full-size automobile cab including windshield, front and rear seating compartments, center console, dash and instrumentation, automatic transmission gear select, two side and one center rear view LCD 
mirrors. The car cab is based on a 2004 Ford Focus. This system provides a flexible and advanced data collection capability for recording vehicle and driver performance measures.

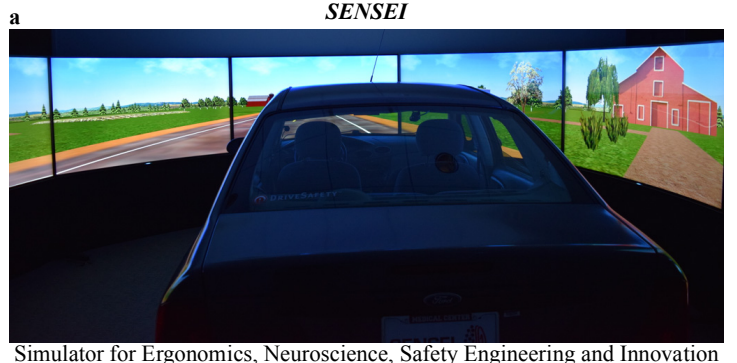

Simulator for Ergonomics, Neuroscience, Safety Engineering and Innovation

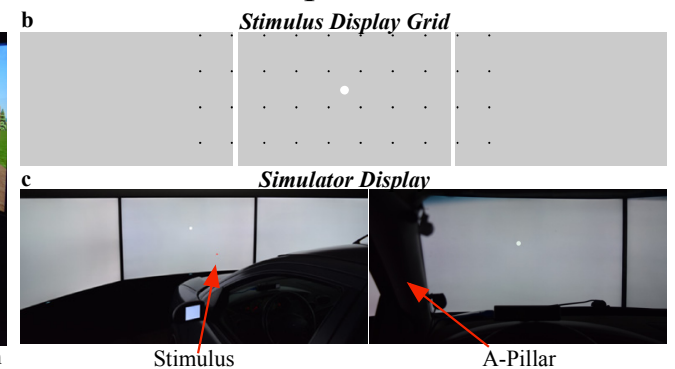

Stimulus

Figure 2. SENSEI and Visual Field Environment

Driving Simulator Visual Field. The Driving Simulator Visual Field (DSVF) was designed to simulate retinal loci in the HVF 30-2 strategy (Figure 2b). Vertical fields were limited by SENSEI display sizes and in-cab geometry (Figure 2c), including obstruction by the A-pillar, precluding direct replication of HVF retinal loci. DSVF maps out grid locations 60 degrees of total horizontal visual angle and 20 degrees of total vertical visual angle, spanning across three displays. Forty imaginary grid locations (10-colums by 4-rows) - each separated by 6 degrees of visual angle across both vertical and horizontal axes - were centered over a fixation dot presented on the center display. Stimulus size subtended a visual angle of 0.5 degrees, similar to the HFA stimulus size III in the HFA. Monocular (left and right) and binocular DSVFs were examined.

DSVF Procedure. Red supra-threshold stimulus images were presented against a light-gray background. Grid locations were tested in random patterns and each grid location was tested 4 times, resulting in 160 serial visual stimulus presentations. Stimulus duration was 200 milliseconds, with a varying inter-stimulus interval (ISI) randomly sampled from a uniform distribution ranging from 1.2 to 1.7 seconds in increments of .017 seconds. Patients were instructed to press a red button on the steering wheel after detecting a stimulus presentation. Hits were counted if the button was pressed prior to subsequent stimulus presentation. Total testing duration was approximately 4 minutes. Each DSVF was repeated twice to ensure reproducibility of scotomas for a total of 6 DSVF tests. Order of testing was randomized.

Data Analysis. Visual field index (VFI) estimates the unimpaired proportion of visual field, ranging between $0 \%$ (fully impaired) to $100 \%$ (fully intact). HVF-VFI estimates were acquired from Humphrey Visual Field Analyzer. The Best Location algorithm was used to integrate HVF monocular visual fields (Nelson-Quigg et al 2000) and a gray scale was used to construct the integrated binocular visual field. Response rates (0-4) and cortical magnification weights were used to calculate a global DS VF-index (DS-VFI; Bengtsson \& Heij1, 2008) for constructed DSVF visual fields. The monocular and binocular gray scales and DS-VFI estimates from the DSVF trials were compared to the gray scales from clinic HVFs.

\section{RESULTS}

One glaucoma patient had unstable visual fields, and was excluded from further analysis. Results from remaining patients $(n=10)$ were all studied. HVF and DSVF measures are displayed in Figure 3. 


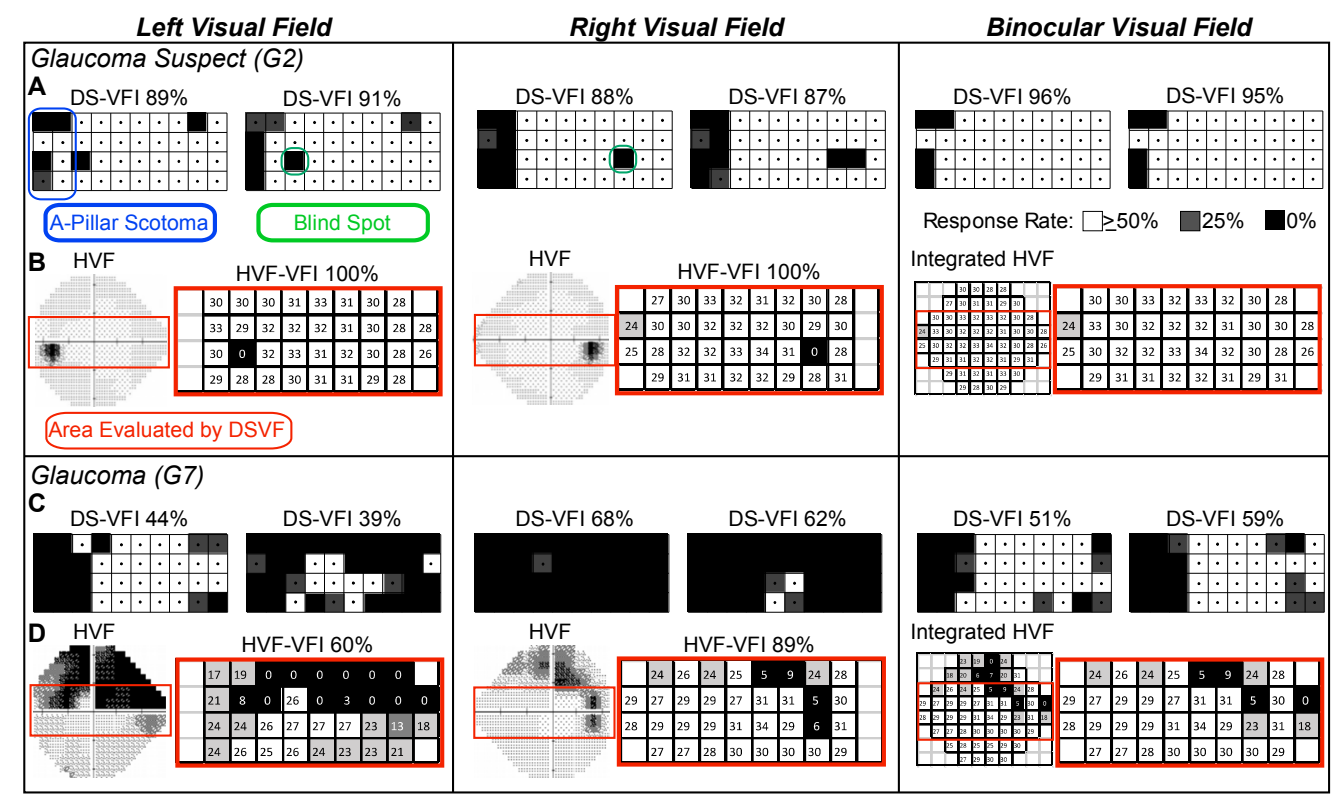

Figure 3. Overview of HVF and DSVF Outcome Measures. Left, right, and binocular visual fields for a selected glaucoma suspect $(\mathbf{A}, \mathbf{B})$ and glaucoma patient $(\mathbf{C}, \mathbf{D})$ are depicted. Visual field performance is summarized by gray scale representations. Each cell depicts performance in each visual field location. In DSVFs (A,C), intensities within each cell correspond to the proportion of trials in which a response was observed. Performance is summarized by the DS-VFI. Visual occlusion by the in-cab A-pillar are apparent in all three visual fields (blue box). Physiologic scotomas imposed by the optic nerve were confirmed in left and right visual fields (green box). In HVFs (B,D), intensities in each cell represent the threshold $(\mathrm{dB})$ values for detecting stimulus presentations. Performance is summarized by the HVF-VFI. For comparison, the 20-degree $\mathrm{x}$ 60-degree region of the HVF evaluated in the DSVF (red box) is provided.

Comparing HVF and DSVF. In glaucoma suspects, monocular HVF-VFI estimates were $>99 \%$ (Figure 3B); DS-VFI estimates were $88.2 \pm 2.9 \%$ (mean $\pm \mathrm{SD}$ ) for left eye, $89.0 \pm 1.0 \%$ for right eye, and $94.9 \pm 1.0 \%$ for binocular visual fields (Figure 3A, 4A). In glaucoma patients, HVF-VFI estimates were $60.7 \pm 23.9 \%$ for worst eye and $88.8 \pm 12.5 \%$ for best eye visual fields (Figure 3D, 5); DS-VFI estimates were $37.6 \pm 32.2 \%$ for worst eye, $58.4 \pm 30.7 \%$ for best eye, and $64.2 \pm 27.8 \%$ for binocular visual fields (Figure 3C, 5). Reductions in DSVF performance relative to HVF performance resulted from visual occlusion by the in-cab A-pillar in the left visual hemifield (discussed below in Artificial Scotomas). Consistent with blind spots mapped in HVF (Figure 3A), DSVF mapped blind spots at $15^{\circ}$ loci (Figures 3B). Indeed, blind spots mapped in all 16 monocular visual fields in glaucoma suspects (Figure 3A, 4A). Estimations of binocular visual fields from HVF (Figure 3B, 3D) were confirmed in DSVF binocular visual fields (Figure 3A, 3C, 5). Split-half reliability (trial-1 vs trial-2) was greater than .99 across left, right, and binocular fields. Absolute DS-VFI differences between each of 2 trials per visual field were $2.2 \pm 1.2 \%$ for glaucoma suspects and $5.8 \pm 5.3 \%$ for glaucoma patients, respectively; furthermore, strong correlations between HVF-VFI and DS-VFI estimates were observed for left $(\mathrm{r}=.77$, $\mathrm{p}<.01)$ and right $(\mathrm{r}=.99, \mathrm{p}<.0001)$ visual fields (Figure 4B). 


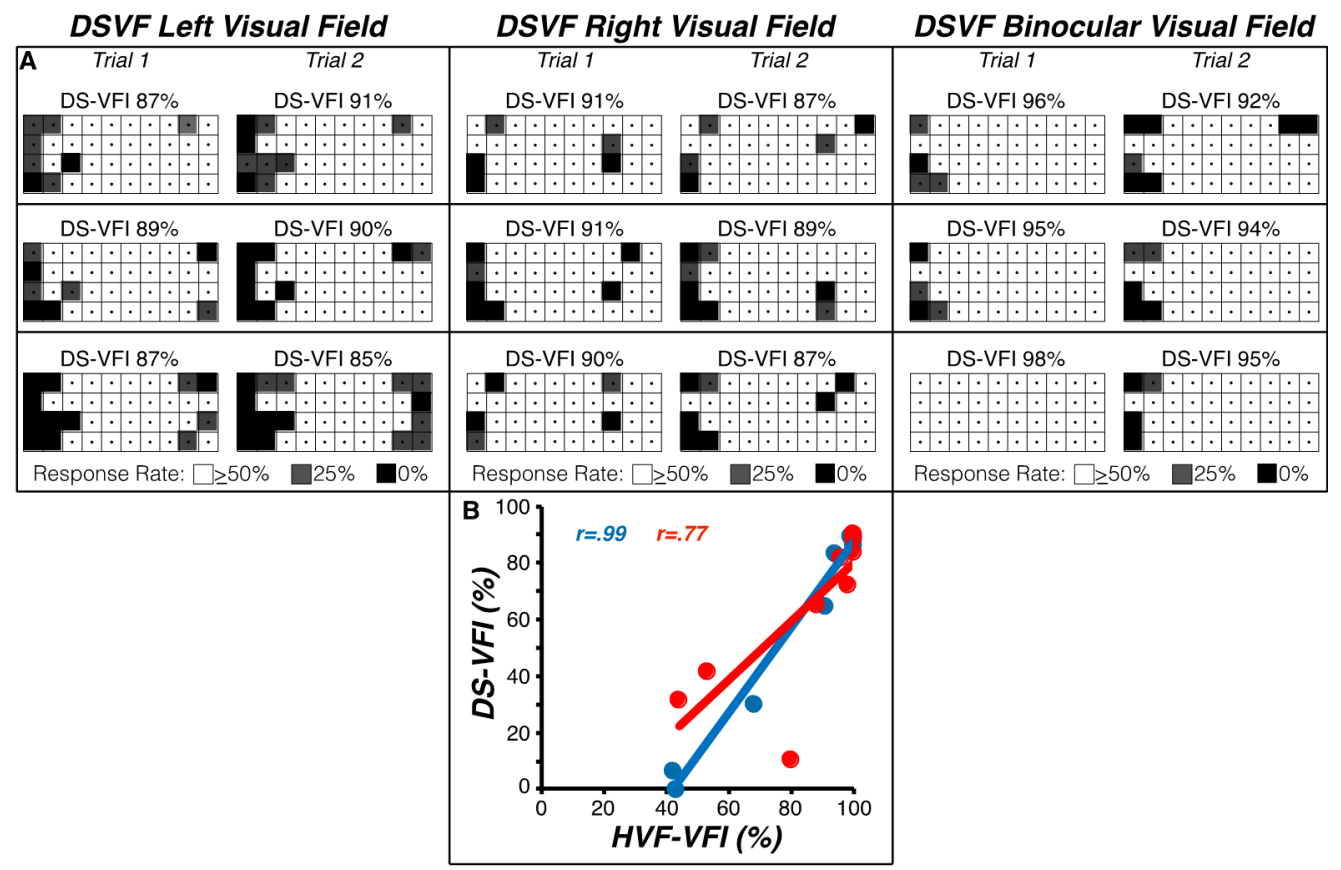

Figure 4: Summary of DSVF in Glaucoma Suspects. (A). DSVF gray scales observed for left, right, and binocular visual fields in three glaucoma suspects. Each trial is presented in separate columns and each glaucoma suspect is presented in a separate row. Gray scale intensitities correspond to the proportion of trials in which a response was observed. DS-VFI values are presented above each DSVF gray scale. (B). Linear regression between HVF-VFI and DS-VFI values observed for left $(\mathrm{r}=.99, \mathrm{p}<.0001 ;$ red $)$ and right $(\mathrm{r}=.77, \mathrm{p}<.01$; blue $)$ visual fields in glaucoma suspects and patients.

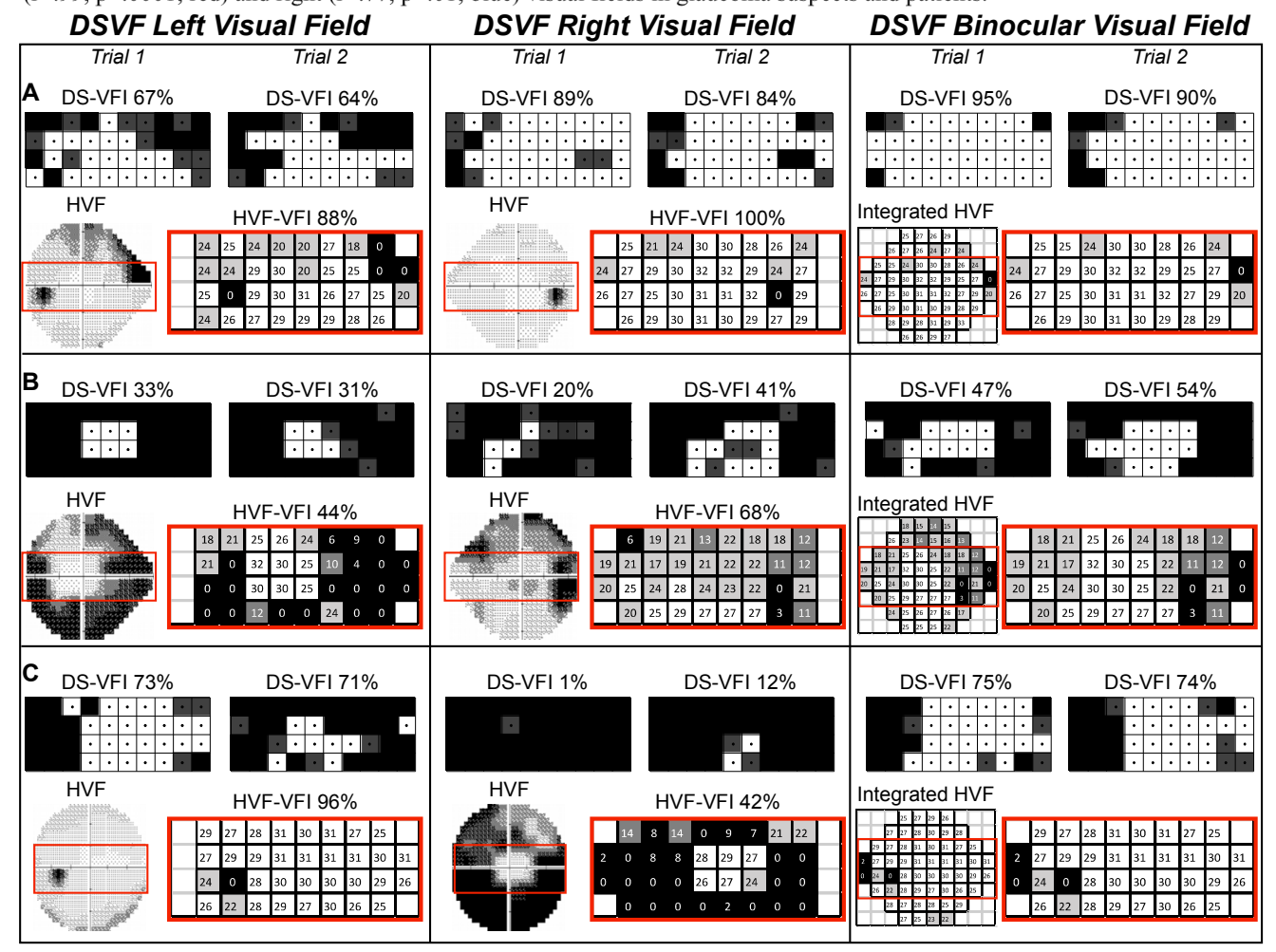

Figure 5: Comparing HVF and DSVF in Glaucoma Patients. (A, B, C). DSVF gray scales observed for left, right, and binocular visual fields in three glaucoma patients. For each glaucoma patient and visual field, DSVF (top) and HVF (bottom) gray scales are provided. DS-VFI values are presented above each DSVF gray scale for first (left) and second (right) trials. Full HVF gray scales (left) are provided with the region tested by the DSVF outlined (red box) and scaled (right) to inspect similarities between measures. 
Artificial Scotomas. In glaucoma suspects, DSVF revealed consistent impairments in stimulus detection at the $21^{\circ}$ retinal locus in the right eye and $27^{\circ}$ locus in the left eye (Figure $3 \mathrm{~A}, 4 \mathrm{~A}$ ) which persists in all 8 binocular visual field trials; in glaucoma patients, impaired stimulus detection is observed across monocular and binocular visual fields (Figure 3C, 5). This apparent visual field defect, corresponding to visual occlusion imposed by the in-cab A-pillar, is approximately $10^{\circ}$ horizontally, reduces binocular VFI by $4.9 \pm .8 \%$, and is unlikely to cause significant functional impairment in normal visual fields. In contrast, glaucoma patients showed a larger reduction in binocular VFI (11.3 \pm 15.9$)$ due to A-pillar occlusion. Thus, glaucoma patients may experience significant impairment as a result of A-pillar occlusion in an already compromised visual field.

\section{DISCUSSION}

Our DSVF task replicated clinical HVFs in a naturalistic driving environment. We successfully mapped physiologic blind spots caused by lack of photoreceptors at the optic disk, peripheral field loss due to optic neuropathy in glaucoma, and estimated binocular visual fields. We observed consistent visual occlusion by the in-cab A-pillar, suggesting vehicle geometry restricts the spatial extent of visual fields in typical populations.

Glaucoma negatively effects driving performance (Crabb et al., 2013; Tanabe, 2011; Kwon, 2016; Owsley et al., 1998; Haymes, 2008). The links between reports of visual experience (Crabb et al., 2013; Hoste, 2003) and physiological effects of optic neuropathy and visual field loss are unclear. The current method offers the opportunity allow us to better understand these links in a naturalistic setting, providing a platform for developing rehabilitation and compensatory strategies to overcome real-world effects of visual field loss.

Visual occlusion by vehicle geometry impairs drivers with normal visual fields and introduces additive impairment to glaucoma drivers with a history of visual field loss. Additional impairments imposed by vehicle geometry during driving may lead to further declines in visual and behavioral function, resulting in reduced driver safety. Further studies are needed to understand how additive visual field loss impairs driving in glaucoma patients and how compensatory behaviors may be developed to overcome these putative limitations.

Fluctuations in visual fields are common and well-studied as in the Ocular Hypertension Treatment Study (Keltner et al., 2000) and were mitigated in this study because our subjects were experienced visual field takers. Variability increases with increasing test sensitivity (Heijl et al., 1989) and the magnitude of visual field defects, and can be reduced by repeating visual fields. Future studies can also combine the DSVF with eye tracking, to map visual fields during driving and determine compensatory effect of eye and head position on field of view.

\section{CONCLUSION}

Our pilot study shows that the DSVF maps out visual fields in participants with and without visual field defects. Field defects (physiologic and occlusive) in the DVSF provide added value in terms of demonstrating ecological effects of visual field defects in operational settings, beyond the impairments demonstrated with conventional clinic-based automated perimetry. The 
occlusive scotoma may have added safety implications in patients with peripheral field loss and may be considered in vehicle safety design. The results of this study may also inform development of in vehicle systems for alerting and warning drivers when obstacles appear in compromised visual fields.

\section{ACKNOWLEDGEMENTS}

We thank the subjects for their participation and patience. This study was supported by a Pilot Grant award from the Department of Neurological Sciences at UNMC and by NIH R01 AG017177.

\section{REFERENCES}

Crabb, D.P., Smith, N.D., Glen, F.C., et al. (2013). How does glaucoma look? Patient perception of visual field loss. Ophthalmology, 120, 1120-1126.

Friedman, D.S., Wolfs, R.C., O'Colmain, B.J., et al. (2004). Eye Diseases Prevalence Research Group. Prevalence of open-angle glaucoma among adults in the United States. Arch Ophthalmol, 122, 532-538.

Haymes, S.A., LeBlanc, R.P., Nicoleia, M.T., et al. (2008). Glaucoma and on-road driving performance. IOVS, 49, 3035-3041.

Hoste, A.M. (2003). New insights into the subjective perception of visual field defects. Bull Soc Belge Ophthalmol, 287, 65-71.

Hu, C.X., Zangalli, C., Hsieh, M., Gupta, L., et al. (2014). What do patients with glaucoma see? Visual symptoms reported by patients with glaucoma. Am J Med Sci, 348, 403-409.

Keltner, J.L., Johnson, C.A., Quiqq, J.M., et al. (2000). Confirmation of visual field abnormalities in the Ocular Hypertension Treatment Study. Arch Ophthalmol, 118, 1187-94.

Kwon, M., Huisingh, C., Rhodes, L.A., McGwin, G., et al. (2016). Association between glaucoma and at-fault motor vehicle collision involvement among older drivers: a population-based study. Ophthalmology, 123, 109-116.

Nelson-Quigg, J.M.,Cello, K., \& Johnson, C.A. (2000). Predicting binocular visual field sensitivity from monocular visual field results. IOVS, 41, 2212-2221.

Owsley, C., McGwin, G., \& Ball, K. (1998). Vision impairment, eye disease, and injurious motor vehicle crashes in the elderly. Ophthalmic Epidemiology, 5, 101-113.

Tanabe, S., Yuki, K., Ozeki, N., Shiba, D., et al. (2011). The association between primary open angle glaucoma and motor vehicle collisions. IOVS, 52, 4177-81. 